\title{
An FDD Multihop Cellular Network for 3GPP-LTE
}

\author{
Rainer Schoenen, Ruediger Halfmann, and Bernhard H. Walke
}

\begin{abstract}
The future cellular radio networks like 3G-LTE [1] are based on an OFDMA physical layer. The duplex scheme is preferably frequency division (FDD) because of its advantages in long range. Huge area coverage in a cost-efficient way is important problem for the early deployment. Time after time the demand in densely populated areas will grow, so a higher cell capacity over the area is needed. The requirements are expensive to solve with a traditional cellular architecture, because a fibre line access will be needed at any base station location, which are sometimes only a few 100 meters apart.

This paper deals with Multihop operation as an option to improve the coverage as well as the capacity issue at low cost. Homogeneous Relays act like Base Stations, but without the need of a cable or fibre access. They are simply fed by the same radio technology in their first hop.

The contribution of this paper is especially the multihop operation in the FDD mode and performance results for the throughput on the MAC-layer. The results were obtained with an analytic model, numerically evaluated with Matlab.
\end{abstract}

Index Terms-FDD, Multihop, Relaying, OFDMA, LTE

\section{INTRODUCTION}

$\mathbf{M}$ ULTIHOP FDD systems have rarely been studied. Relaying has been studied mostly for TDD systems [2]. But multihop capable air interfaces are feasible for both duplex schemes and provide their benefit because of the improved coverage and capacity in a cell.

While there is always demand for high data rates almost homogeneously over the area, conventional cellular architectures cannot offer this exactly. Due to the limited transmit power (EIRP limited), the higher transmission rates lead to a lower energy per bit. The radio propagation at higher frequencies is vulnerable to bad non-line-of-sight conditions. In effect, the path loss is higher between base station (BS) and user terminal (UT). Also the offered rate is not homogeneous over the area. The maximum data rate offered by a BS depends on the distance of the mobile to the base station. Close to the BS, the higher received SINR value allows the highest Modulation\&Coding scheme (PhyMode), which results in the highest data rate. At the cell border the offered data rate is one order of magnitude lower (QPSK $\frac{1}{3}$ compared to $Q A M 64 \frac{5}{6}$ for LTE [1]). What makes this even worse is that a transmission operating with the lowest PhyMode occupies a ten times higher part of the spectrum than a transmission using the highest PhyMode. That means the average cell capacity is overproportionally determined by the maximum possible rate at the outer regions.

More base stations per area is one approach, but this comes with much higher deployment costs, since every base station

\footnotetext{
- Ruediger Halfmann is with Siemens, Munich, Germany

- The other authors are with the Chair of Communication Networks at RWTH Aachen University, Faculty 6, Germany

- This work has been funded by the BMBF in Germany in the ScaleNet project
}
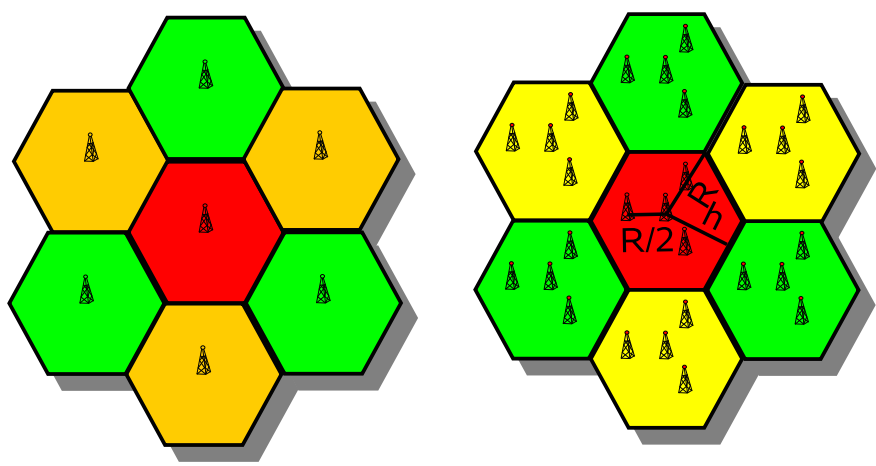

Fig. 1. Left: Singlehop cellular geometry; Right: Multihop cell with increased capacity. Here a cluster order of 3 is shown.

needs its own access to the fiber backbone network. Alternatively we can deploy Fixed Relay Stations, also called Relay Nodes (RN), that are fed over the same wireless technology. The goal of this is the almost ubiquitous provision of very high data rates for any user terminal within the cell. Figure 1 shows the two ways from a conventional cellular layout to multihop-augmented cells for both goals.

The OFDM transmission scheme allows the optimum use of radio resources, especially if multiple access is possible within a frame (OFDMA). OFDMA scheduling determines the parameters to use for each resource unit [3], i.e. choosing the best subcarrier for a user terminal, setting the PhyMode to use and controlling the RF power per subcarrier. In this paper we concentrate on FDD (preferred in huge area cellular networks) and address the MAC frame organization, relay enhanced extensions and performance results for the upcoming long term evolution (LTE) technology [1].

The paper is organized as follows. The first section defines the relaying operation and their properties. Next, the additional opportunities provided by orthogonal frequency division multiple access (OFDMA) are explained. The principles of the FDD mode operation is explained in section IV. The last section presents performance results for capacity and coverage increase scenarios.

\section{Multihop Operation}

We consider here decode-and-forward or layer-2 schemes, where relays act as repeaters, bridges or routers. In these systems, received PDUs are fully error-corrected, automatically repeated (ARQ), stored and scheduled for transmission, and if necessary even segmented and reassembled prior to their next-hop transmission.

Multihop systems are transparent for the UT. The RN acts like a BS towards the UT on the second (last) hop. Only for the first hop(s), where the RN acts like a UT towards the BS, 


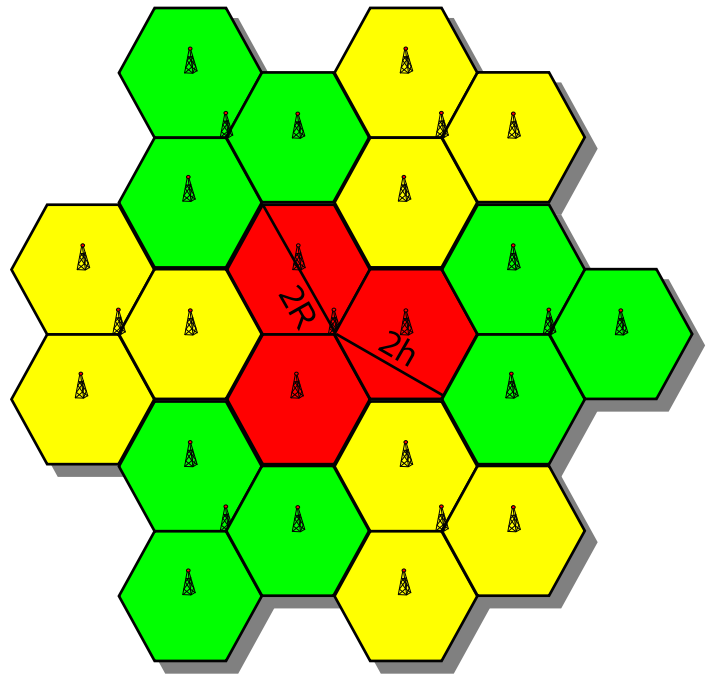

Fig. 2. Multihop cellular geometry with increased coverage area. Here a frequency reuse factor of 3 is shown.

additional resources are required. It depends on the multiple access scheme what we are thinking of when using the word 'resources'. While for CDMA the resources are orthogonal code channels, in TDMA we have time frames (resources of $\mathrm{BS}$ and RN can be separated in time) and in FDMA we have subchannels. In OFDMA there is luckily a combination of both. Relaying was first introduced only for TDM systems [4].

At ISO-OSI layer-2, the medium access control (MAC) is in charge of introducing the relay resources into the frame structure of an existing protocol. For relaying we basically have the (transparent) subframe concept [5] and the (relayaware) resource reservation concept which we follow here. For this, a multihop transmission is coordinated by centralized control located in the base station.

\section{A. Scenarios}

Relaying means any traffic destined from the fixed network towards the UT goes through BS and one or more RNs. We assume one relay (two hops) now, but there is no principal limitation in the number of hops. The traffic is packetized in protocol data units (PDUs) which are transmitted by the BS to the RN (first hop, hop 1 ) using dedicated downlink (DL) resource blocks. In the $\mathrm{BS}$, the $\mathrm{RN}$ is treated like a UT. For the next hop, the PDUs require resources (sending opportunities) between RN and UT in the downlink. These resources are assigned by the BS which is relay aware. So the BS has reserved DL resources which it doesn't use itself, but lets the RN operate on it. The RN has full freedom of use for these resources. It decides like a BS which resource to use for which of its UTs. The size of the resources for the second hop may differ from that of the first hop (if the $S I N R$ situation is different), so different PhyModes are used. This fact has to be taken into account when reserving resources for hop2. UTs receive PDUs from RN just like they would from a BS. They do not see a difference and do not need to be relay aware for that purpose.

For the uplink (UL), an UT announces any available traffic

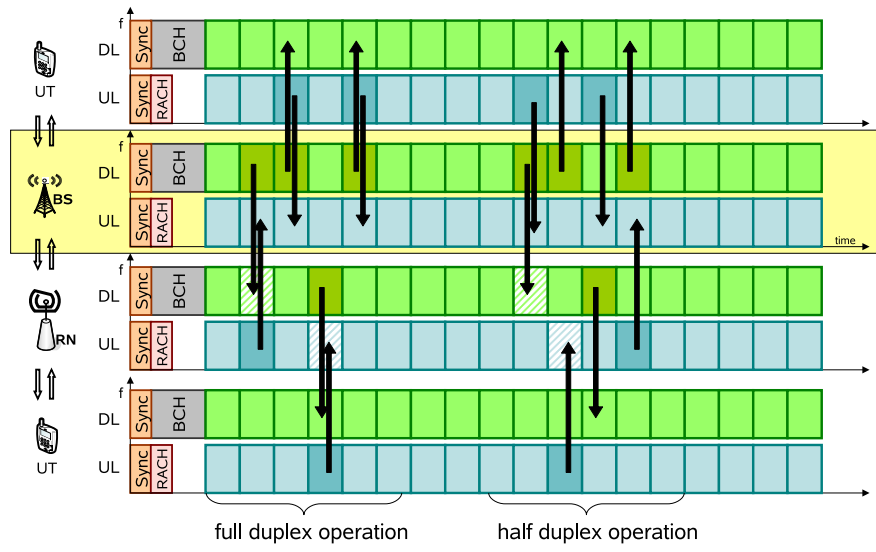

Fig. 3. Radio Frame format for FDD operation. Frame at BS: Base Station sends in downlink and receives in uplink resources. Top and bottom frame: User Terminals send in uplink and receive in downlink resources. Frame 3: Relay Node has transceivers that can send+receive both in UL and DL

to its master station, which is the RN. The RN assigns UL resources to UT for the PDU transmission while it also announces this new uplink traffic towards the BS. PDUs received in the uplink of hop 2 are stored in $\mathrm{RN}$ until the UL resources are available for hop 1 . So the relaying problem simplifies to the control of resources, which is a task of any base station controlled (central) cellular system anyway.

Typical scenarios for cellular multihop operation are shown in figure 1. In the coverage extension scenarion, the supported area (regarding sufficient $S I N R$ ) around relay nodes is of same size as the singlehop area around the base station. The inner hexagon is typically served by BS within its range, while the outer regions are served by one of the three RNs respectively. For capacity extension, the whole hexagonal area can be served by the BS, but in regions closer to the cell border and near the $\mathrm{RN}$, the $\mathrm{RN}$ offer a much higher data rate to user terminals than the BS would.

Being served by a RN means that the decision has been taken that the UT is better supported by the RN than by the BS. 'Better' not only means that the UT receives a higher $S I N R$ at its current position, but additionally that the total amount of resources needed for hop 1 (BS-RN) and hop 2 (RNUT) is less than what would be required if it was a single hop transmission between BS and UT. It means that at this position relaying improves the spectral efficiency of the cell.

\section{OFDMA RELAYING}

While relays are possible for all kinds of transmission schemes, OFDM has evolved as an efficient multiplex scheme of typically 1024 small orthogonal subcarriers within the system bandwidth. Small subcarriers mean long symbols in time, so the problem of inter symbol interference (ISI) is relieved. In the classical OFDM, symbols are not subdivided among different users, i.e. one OFDM symbol is always completely assigned to one user. The big advantage of OFDM is that each subcarrier can have a different PhyMode. So a robust BPSK can be used on frequencies where the channel is currently bad due to fading, while e.g. QAM64 can be used on more stable subcarriers with higher $S I N R$ values. 


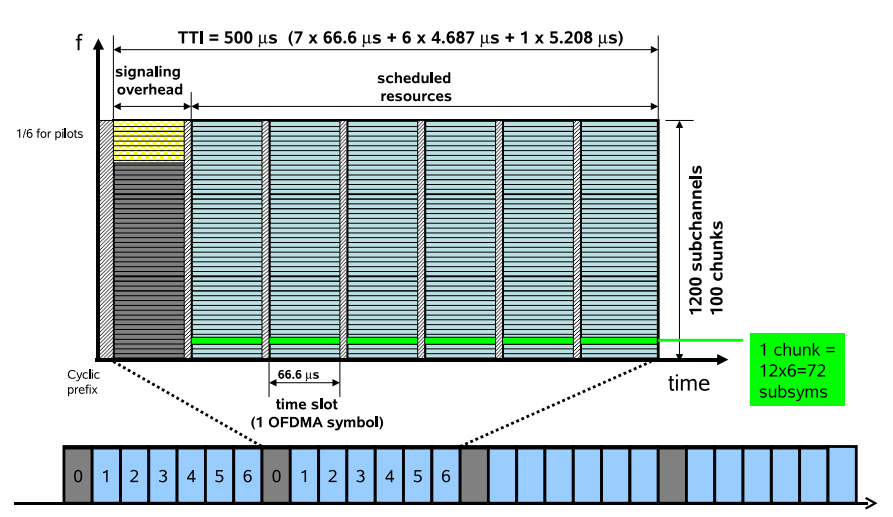

(a) One Downlink TTI frame

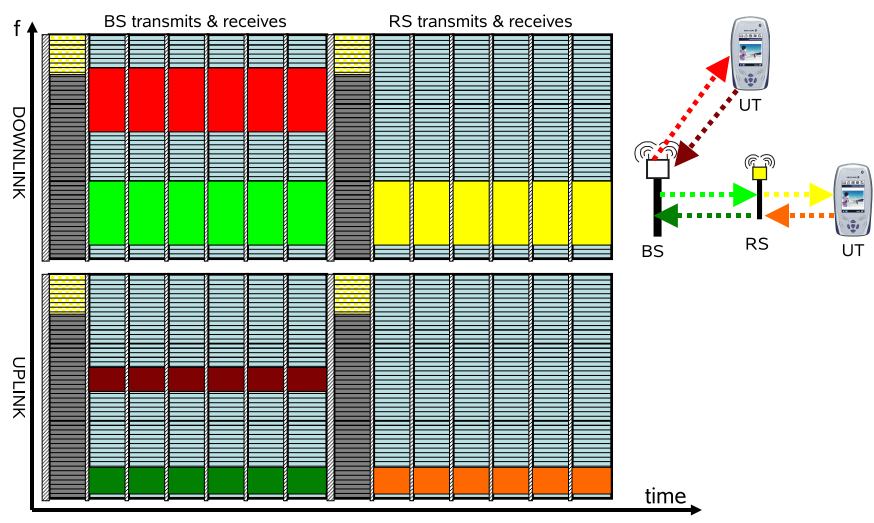

(b) DL and UL frames for relaying

Fig. 4. Frame format for OFDMA transmission. This example shows one TTI frame $(500 \mu s)$ for 3GPP-LTE [1]. On the right, a dynamic resource allocation for a relaying scenario is shown.

Channel coding can also be adjusted in wide ranges to adapt to the subchannel conditions. This is called dynamic adaptive modulation and coding (AMC) [3].

OFDMA means that within one OFDM symbol, several different UT receivers can be addressed. So for example, 256 subcarriers are used for each of four UTs that receive the symbol. Dynamic subcarrier assignment (DSA) selects the best resources for each UT based on channel state information (CSI). As the distance and pathloss may be very different among the UTs, it is likely that the PhyModes used for each UT are very different within the full OFDM symbol.

In OFDMA, the granularity of these resources is typically a chunk, i.e. a group of subcarriers if $f$-direction and a number of OFDM symbols in $t$-direction. Figure 4(a) shows the frame format proposed for 3GPP-LTE [6]. A chunk of size 12 subcarriers times 6 effective symbols (gross 7 ) can carry up to 360 bits in PhyMode $64 Q A M-5 / 6$. Resources used at one location (cell) can be reused at another place (see frequency reuse distance $=$ cluster order 3 in figure 1 left). But also within one cell, there can be a spatial reuse by using beamforming, multipath transmission, spatial multiplexing by the use of distributed antenna elements [7].

\section{FDD TIME-DOMAIN RELAYING}

For relaying without intra-cell interference there must be orthogonal resources (resource blocks in $f$ and $t$ ) assigned to each UT and RN by the BS. The duplex mode, i.e. how to separate uplink from downlink traffic, is simply another use of orthogonality. In time division duplex (TDD), downlink and uplink phases alternate periodically [8], requiring a guard time between phases. The downlink to uplink ratio can slowly be adapted e.g. between $5: 1,3: 3$ and $1: 5$ as there are six OFDM symbols per frame. For multihop from time to time a complete frame is allocated to the second hop, so that the packet transport over the wireless loop has four phases for which it takes four frames: $B S \rightarrow R N, R N \rightarrow U T$, $U T \rightarrow R N$ and $R N \rightarrow B S$.

FDD mode relaying shares the advantages of FDD in the singlehop case, i.e. no need for guard times and less interference for DL and UL transmissions. The BS always sets up the master frame, i.e. generates the timing schedule for the next period. Beginning with synchronisation pilots, a broadcast channel $(\mathrm{BCH})$ and a random/contention access channel (RACH), a number of regular frames follow which depend on the technology, see Figure 3. The BS also reserves some of the frames for the second hop(s), to be used in the responsibility of the relay(s). Figure 3 also shows the case of half duplex UTs, which in contrast to full duplex UTs cannot receive and send at the same time. This saves expensive rf duplex filters in the user equipment and is easy to incorporate [9].

In Figure 4(a) the frame format shows the smallest granularity resource unit (chunk) of which 100 fit into the bandwidth. Symbols used for signaling and synchronization are out of focus here (shown in grey) and the only impact here is some overhead (1/7). Downlink and uplink transmissions happen simultaneously (FDD) in the same frame raster. Relay resource partitioning, i.e. how to coordinate the frames used for the second hop (or beyond) can be done either in time or frequency domain [2]. Since frequency domain relaying needs more total system bandwidth, we focus on time domain relaying here.

\section{A. Time Domain Relaying}

Figure 4(b) shows the store-and-forward way of relaying where one frame is used to serve hop- 1 users plus the relay(s), and another frame is dedicated to the hop- 2 users. The BS uses dedicated resources on the downlink channel for the first hop which it can allocate for itself, knowing the traffic demand. Transmissions to and from the RN happen in the same frame, just like with any UT. The traffic is known as well because the downlink scheduler knows the number of packets in its queues.

For the second hop DL transmissions, the RN is actively transmitting. Because RN and BS are hard to synchronize, especially for the required OFDM orthogonality, all the power of the other sender must be treated as side-band interference. So the transmission must happen in a separate frame to avoid interference and the BS must reserve the complete resources of this frame for the second hop. 
The frame schedule at BS,RN,UT1,UT2 for a small scenario is shown in figure 3. In the downlink direction either the BS sends to UTs and RNs, or the RN uses this time slot. The uplink is simply used in parallel at the same time, but this is not necessarily required.

With OFDMA, the resources can be subdivided in a finer granularity than what would be possible with OFDM only. Figure 4(b) shows that first-hop transmissions are all treated the same way. They just occupy the required resources for their traffic. In the uplink also several UTs share the full bandwidth, each of them transmitting on a subset of subchannels, just like in the DL. The BS or RN coordinates the orthogonal noninterfering use of these subchannels by the UTs.

\section{Multihop Performance}

The Figures 1 and 2 show the scenarios that were studied for relaying of 3G-LTE. The analytical model used for the analysis is based on a closed formula for $S I N R$ to $M I$ [10], which expresses the the link level function [11] between $S I N R$ and sustainable rate for each PhyMode. The results were then obtained numerically for each position in the area by the use of Matlab.

\section{A. MAC Layer Analytical Model}

From link level to MAC throughput, the performance of the example system is evaluated according to these steps:

- Transmit Power: $37 \mathrm{dBm}$ at the BS, $34 \mathrm{dBm}$ at the RN,

- Bandwidth: $b=18 \mathrm{MHz}$ net (20MHz system),

- Frequency: $2.1 \mathrm{GHz}$ apropriate for LTE,

- Pathloss I: non-line-of-sight propagation,

- Pathloss II: slow and fast fading effects [12],

- Antenna Gain: BS to RN antenna gain is $14 d B$ due to stationarity (single antenna assumed),

- Interference: neighbor cell BSs interfere (100\% load, cluster order $C=7$ ),

- Noise: accounted for but not serious in interferencelimited systems,

- SINR: the first performance measure below PHY layer,

- MI: mutual information [13] determined from SINR and modulation as described in [10],

- BER: bit error ratio, the PHY performance result, depends on the Channel coder used,

- PER: packet error ratio, the result after channel decoding $\left(P E R=1-(1-B E R)^{N / b i t s}\right)$,

- Delay: determined by PER (ARQ retransmissions) and roundtrip times $\left(d_{\text {above } A R Q}=T_{\text {frame }} \cdot(1-P E R)^{-1}\right)$,

- Throughput: determined by bandwidth, PhyMode (modulation and code rate) and ARQ overhead ( $r_{\text {above } A R Q}=$ $\left.r_{\text {below } A R Q} \cdot(1-P E R)\right)$.

- Second Hop Throughput: reduced by resources required on first hop $\left(r_{2}=\left(r_{1, \max }^{-1}+r_{2, \max }^{-1}\right)^{-1}\right)$.

\section{B. Analytical Results}

The steps in section $\mathrm{V}$ are evaluated for each location in the scenario area. Interference from neighbor cells (cluster order 7) is accounted for. The coverage increasing scenario
(Fig. 2) results in DL $S I N R$ and rate performance as shown in Figure 5 above. The capacity increasing scenario (Fig. 1) performs as shown in Figure 5 below. SINR results here show the $S I N R$ of the best serving station (BS,RN), not the maximum $S I N R$. The best station is defined as the one offering the highest data rate to the UT. The data rate results mean the maximum achievable rate at a certain position within the cell, taking also the required first hop resources into account. Therefore, the second hop maximum rate near the $\mathrm{RN}$ cannot be as much as near the BS. In all cases, the RN is only chosen as the serving station if this is an advantage for the cell, i.e the least resources are used. In both scenarios there are huge areas where the relay offers an advantage over the singlehop case. So relaying offers more than just higher $S I N R$ areas around RNs. In contrast to the analytic approach here, simulation results can be found in [14].

\section{Coverage Extension}

In Figure 2 the cell geometry is extended so that the covered area is three times the original area $(+200 \%)$. In figure 5(a) (top) the area served by the BS is small compared to the coverage achieved by the relays. Figure 5(b) shows that the maximum rate around the RNs is only half of the BS rate, but in areas the BS would never cover. So it can be used to extend the coverage very economically, while the inner cell still has capacity to offer.

\section{Capacity Extension}

For capacity extension, RNs are placed within the normal radius of the single-hop cell (Figure 1), i.e. even without RNs the BS is able to provide coverage for all UTs in the cell. But with additional RNs there are areas near the cell border where a RN provides much better service, not only in terms of $S I N R$ (fig. 5(c)) but also for the total offered capacity (fig. 5(d)). By this way the cell border is no longer an area of the worst PhyMode, but instead gets a quite high PhyMode. Altogether, two transmissions with both high PhyMode are better than one transmission hop with a bad PhyMode.

\section{CONCLUSION}

This paper treats the properties and performance of multihop FDD mode cellular systems using OFDMA. Especially with OFDMA there is a great flexibility to fine-tune resource consumption and interference mitigation. Two scenarios, the Coverage Extension Scenario and the Capacity Extension Scenario, are analyzed for multihop cellular networks. It is shown that for each scenario the multihop communications can provide an remarkable increase in layer- 2 capacity. Especially in areas suffering from high path loss, at the cell border or around obstructions, relays can be successfully applied. The economic benefit is the fact that no wired line access is required and relay devices can be cheaply installed anywhere. The benefit is also that the high capacity near the BS can be traded against coverage or better capacity at the outer regions.

Future work in progress is the detailed simulative evaluation with realistic resource schedulers and timing. 


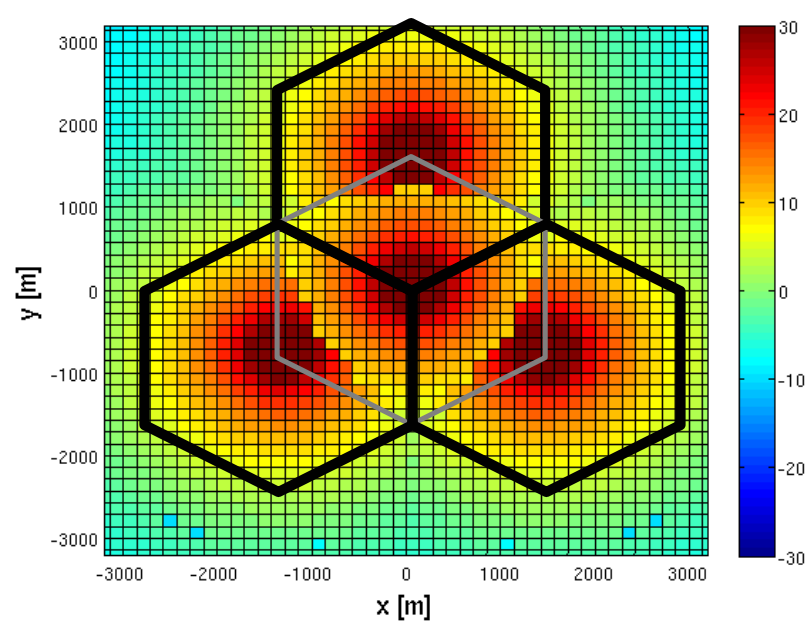

(a) Coverage Extension Scenario: SINR(area) in $\mathrm{dB}$

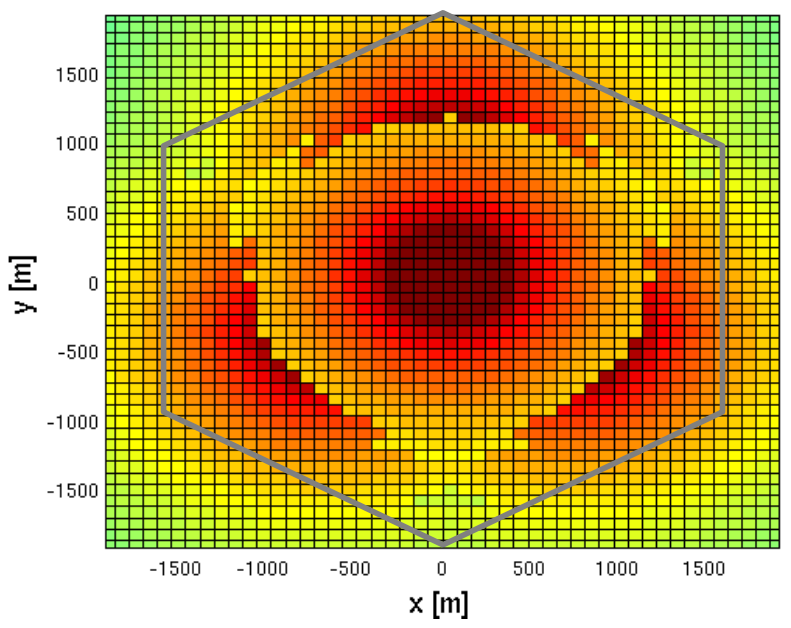

(c) Capacity Extension Scenario: SINR(area) in dB

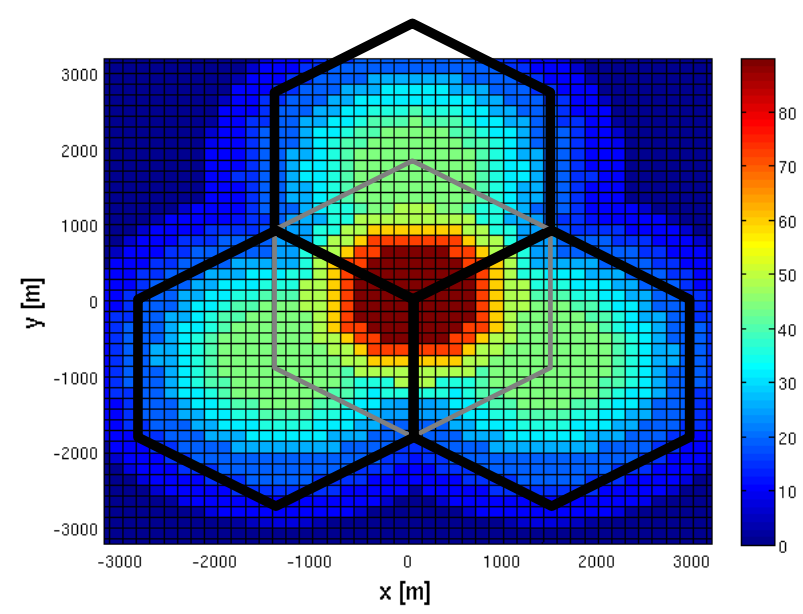

(b) Coverage Extension Scenario: Rate(area) in Mbit/s

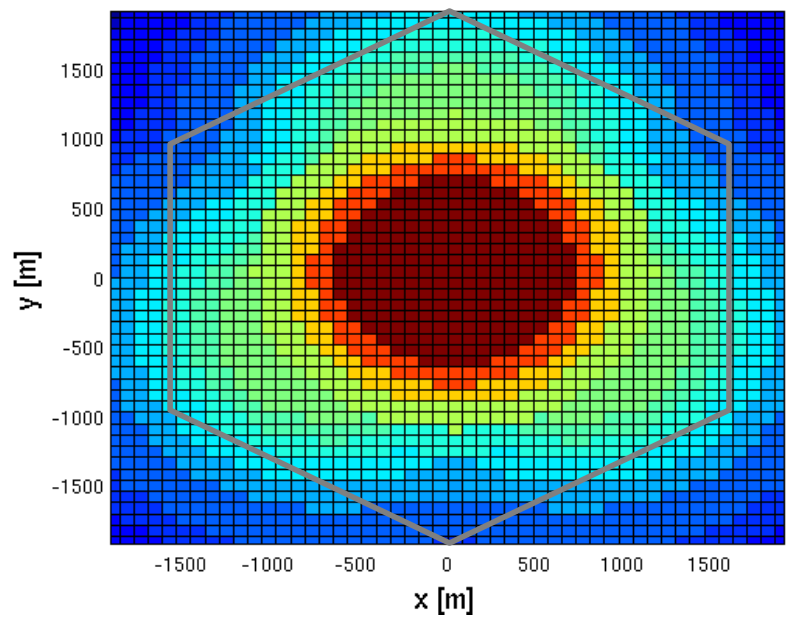

(d) Capacity Extension Scenario: Rate(area) in Mbit/s

Fig. 5. Results for the two basic relaying scenarios. Values are for 3GPP-LTE, but the principles are the same for other technologies. In the graphs, a DL capacity close to $100 \mathrm{Mbit} / \mathrm{s}$ in the center is achieved in both scenarios.

\section{REFERENCES}

[1] http://www.3gpp.org/Highlights/LTE/LTE.htm.

[2] R. Pabst, B. Walke, D. C. Schultz, and et al, "Relay-Based Deployment Concepts for Wireless and Mobile Broadband Radio," IEEE Communications Magazine, pp. 80-89, Sep 2004.

[3] G. Song and Y. Li, "Utility-based resource allocation and scheduling in OFDM-based wireless broadband networks," IEEE Communications Magazine, pp. 127-134, Dec 2005.

[4] B. Walke and R. Briechle, "A local cellular radio network for digital voice and data transmission at 60ghz," in Proceedings Cellular \& Mobile Comms. Intern. London: Online, Nov 1985, pp. 215-225. [Online]. Available: http://www.comnets.rwth-aachen.de

[5] N. Esseling, H. Vandra, and B. Walke, "A forwarding concept for hiperlan/2," in Proceedings of European Wireless 2000, Sep 2000, pp. 13-18. [Online]. Available: http://www.comnets.rwth-aachen.de

[6] K. J. e. a. Ekstrom H., Furuskar A., "Technical solutions for the $3 \mathrm{G}$ long-term evolution," IEEE Communications Magazine, pp. 38-45, Mar 2006.

[7] E. Zimmermann, P. Herhold, and G. Fettweis, "On the performance of cooperative diversity protocols in practical wireless systems," in 58th Vehic. Techn. Conf., Orlando, FL, Oct 2003.

[8] W. Mohr, "The WINNER (Wireless World Initiative New Radio) Project - Development of a Radio Interface for Systems beyond 3G." in Proc. of IEEE Personal Indoor and Mobile Radio Conference 2005 (PIMRC05), Berlin, Germany, Sep 2005.
[9] A. Otyakmaz, R. Schoenen, and B. Walke, "Parallel Operation of Halfand Full-Duplex FDD in Future Multi-Hop Mobile Radio Networks," in Submitted to the European Wireless Conference, Prague, Jun 2008.

[10] R. Schoenen and B. Walke, "On PHY and MAC performance of 3G-LTE in a multi-hop cellular environment," in Proceedings of the 3rd IEEE International Conference on Wireless Communications, Networking and Mobile Computing (WiCOM), Shanghai, China, Sep 2007. [Online]. Available: http://www.comnets.rwth-aachen.de

[11] M. Ahmed, H. Yanikomeroglu, and S. Mahmoud, "Fairness enhancement of link adaptation techniques in wireless access networks," in Proc. Vehic. Technol. Conf.-Fall, 6-9 Oct. 2003, pp. 1554-1557.

[12] M. Einhaus, O. Klein, B Walke, and R. Halfmann, "Simulative mac level performance evaluation of an ofdma system under the consideration of frequency correlated fading," in Proceedings of the 2007 IEEE Wireless Communications and Networking Conference, Hong Kong, Mar 2007, p. 6. [Online]. Available: http://www.comnets.rwth-aachen.de

[13] K. Brueninghaus and D. e. a. Astely, "Link performance models for system level simulations of broadband radio access systems," in Proceedings of the 17th Annual IEEE International Symposium on Personal, Indoor and Mobile Radio Communications, Sep 2005, pp. 2306-2311.

[14] N. Esseling, R. Pabst, and B. Walke, "Delay and throughput analysis of a fixed relay concept for next generation wireless systems," in Proceedings of 11th European Wireless Conference 2005, vol. 1, Nikosia, Cyprus, Apr 2005, pp. 273-279. [Online]. Available: http://www.comnets.rwth-aachen.de 